\title{
On the Bits per Joule Optimization in Cellular Cognitive Radio Networks
}

\author{
Wuchen Tang, Muhammad Z. Shakir, Khalid A. Qaraqe, Erchin Serpedin, \\ Muhammad A. Imran, and Rahim Tafazolli
}

\begin{abstract}
Cognitive radio has emerged as a promising paradigm to improve the spectrum usage efficiency and to cope with the spectrum scarcity problem through dynamically detecting and re-allocating white spaces in licensed radio band to unlicensed users. However, cognitive radio may cause extra energy consumption because it relies on new and extra technologies and algorithms. The main objective of this work is to enhance the energy efficiency of proposed cellular cognitive radio network (CRN), which is defined as bits/Joule/Hz. In this paper, a typical frame structure of a secondary user (SU) is considered, which consists of sensing and data transmission slots. We analyze and derive the expression for energy efficiency for the proposed CRN as a function of sensing and data transmission duration. The optimal frame structure for maximum bits per joule is investigated under practical network traffic environments. The impact of optimal sensing time and frame length on the achievable energy efficiency, throughput and interference are investigated and verified by simulation results compared with relevant state of art. Our analytical results are in perfect agreement with the empirical results and provide useful insights on how to select sensing length and frame length subject to network environment and required network performance.
\end{abstract}

Index Terms-Cellular cognitive radio networks; energy efficiency; throughput; spectrum sensing; optimal frame structure.

\section{INTRODUCTION}

Spectrum efficiency is an critical issue for cellular communication networks. However, recent reports by Federal Communications Commission (FCC) have pointed out that $70 \%$ of the allocated spectrum bands in the US is not fully utilized, whereas only $2 \%$ of the spectrum is used in the US at any given moment of time. [1]. Cognitive radio was first introduced in 1999 [2] to improve the current severely underutilized radio spectrum by allowing the unlicensed secondary user (SU) to access the frequency bands licensed to primary user (PU) [3-5].

\section{A. Motivation}

In addition to spectrum efficiency enhancement as the primary target, another important issue for the sustainable development of cognitive radio technology for cellular networks is energy efficiency, which has been recently marked as one of the alarming bottlenecks in the telecommunication growth paradigm mainly due to dramatically varying global climate [6] and slowly progressing battery technology [7]. Therefore, currently green radio is playing an equally or even more important role than increasing the communication

W. Tang, M. A. Imran and R. Tafazolli are with Centre for Communication Systems Research (CCSR), University of Surrey, Guildford, Surrey, United Kingdom, GU2 7XH, email:\{w.tang, m.imran, r.tafazolli $\}$ @ surrey.ac.uk

M. Z. Shakir and K. A. Qaraqe are with the Electrical and Computer Engineering Dept., Texas A\&M University at Qatar, Doha, Qatar, email: \{muhammad.shakir, khalid.qaraqe\}@qatar.tamu.edu.

E. Serpedin is with the Dept. of Electrical and Computer Engineering, Texas A\&M University, TX, USA, email: serpedin@ece.tamu.edu.

This publication was made possible by NPRP grant\# 4-1293-2-513 from the Qatar National Research Fund (a member of Qatar Foundation). The statements made herein are solely the responsibility of the author[s]. capacity and has prompted new waves of research and standard development activities. High energy efficiency will be one of the key requirements for practical deployment of cellular cognitive radio networks (CRNs). However, the majority of the current research still aims at improving the throughput of CRN [8-10] while energy-efficiency issues in CRN have not been well addressed until recently when some works have started to touch. In [11], the optimal sensing strategy is studied based on sequential sensing over multiple channels. The sensingaccess strategies and the sensing order are identified to achieve the maximum energy efficiency. However, only the variable sensing length is considered. In [12], the authors proposed energy efficient transmission duration design and power allocation methods. However, the considered system model is very simple and the interference from PU to SU is ignored. Because the length of sensing slot is assumed fixed, the impact of the sensing length on the sensing accuracy is not considered.

\section{B. Main Contribution}

In this paper, we investigate the variable frame structure of SU which consists of sensing and data transmission slots. The energy efficiency (bits/Joule/Hz) in the proposed cellular $\mathrm{CRN}$ is formulated as a function of variable sensing and data transmission slot durations under practical primary traffic load. The impact of optimal sensing time and frame length on the achievable energy efficiency, throughput and interference is investigated and studied. The accuracy of derived theoretical expressions is compared with relevant state of art and verified by simulation results. The selection of optimal sensing and data transmission slot durations of the SU cognitive transmission with different performance metrics, e.g, energy efficiency and throughout, under a practical traffic load environment is addressed and the diversity is highlighted in a cellular CRN set-up.

The rest of this paper is organized as follows. Section II defines the system model. In Section III, the problem of energy efficiency optimization is formulated followed by the analysis based on the frame structure. Performance analysis and discussions to validate the analytical results are presented in Section IV followed by the concluding remarks in Section $\mathrm{V}$.

\section{SYSTEM MODEL}

In this paper, we consider the uplink of a single secondary system coexisting with a primary system in a cellular CRN. The cognitive data transmission of the SU is activated subject to the spectrum sensing outcomes. A typical frame structure of the SU is shown in Fig. 1 where the frame of length $T$ consists of the sensing slot with length $\tau$ and the data transmission slot with length $T-\tau$. The SU performs energy detection [13] for spectrum sensing and then decides to transmit or keep silent according to the sensing result. Once the channel has been confirmed as idle, the SU starts to transmit on the corresponding channel because we assume (i) the SU is heavily loaded and always has data to transmit due to its secondary priority, (ii) 


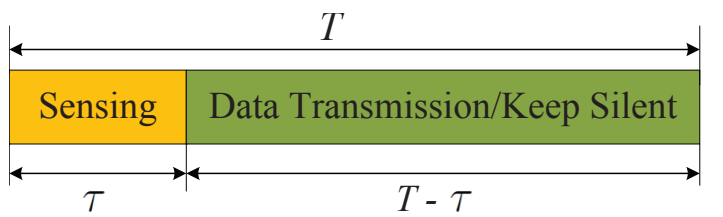

Fig. 1. Graphical illustration of a typical frame structure of a SU cognitive radio transmission.

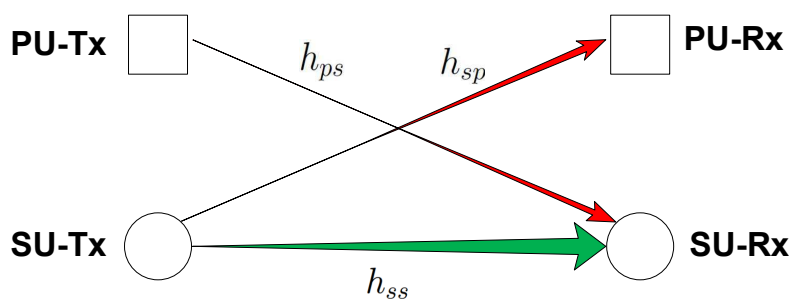

Fig. 2. Graphical illustration of transmission and interference links.

the traffic load of the PU is exponentially distributed with the mean of the occupied and the idle durations denoted by $\alpha_{1}$ and $\alpha_{0}$, respectively.

Any single primary and secondary transmission on the available channel consists of a transmission link and two interference links, as depicted in Fig. 2, where Tx and Rx denote the transmitter and receiver, respectively. The instantaneous channel gain of the secondary transmission link on the available channel is denoted as $h_{s s}$ while the interference link from PU to SU and from SU to PU are shown as the narrow arrows with channel gains $h_{p s}$ and $h_{s p}$, respectively. Channel is assumed ergodic stationary and known before the transmission phase. The quality of channel could be estimated in a very short time interval compared with the sensing length. For example, in IEEE 802.11a, only 4 pilot symbols are used for channel estimation [14] while in IEEE 802.22 wireless regional area network (WRAN) thousands of samples are required for a typical sensing [8]. Therefore, for the frame structure of $\mathrm{SU}$, the channel estimation time could be ignored and thus is not considered in Fig. 1. The signals of PU are assumed to be complex-valued phase-shift keying (PSK) signals, whereas the noise at the SU is assumed to be independent and identically distributed circularly symmetric complex Gaussian (CSCG) with zero mean and $N_{0}$ variance.

It is noted that even if the channel is sensed and is available for SU to transmit, the corresponding PU might still become activated during the data transmission phase of the current frame. This would lead to the situation that both the SU and the PU transmit on the same channel which not only decreases the throughput of SU but also introduces interference to the PU. In this case, the percentage of transmission with interference due to this PU unpredictable activity out of data transmission duration could be expressed as [15]

$$
P_{p}^{s}=1-\frac{\alpha_{0}}{T-\tau}\left(1-\exp \left(-\frac{T-\tau}{\alpha_{0}}\right)\right) .
$$

In addition, there may be additional interferences because of sensing errors of the spectrum, that is, the situation where the spectrum is actually occupied by the PUs and wrongly detected as idle which is referred to as missed detection. In practice, spectrum sensing is always imperfect and the sensing errors due to missed detection lead to more interference between the SU and PU. In the situation that missed detection occurs, the percentage of transmission with interference out of data transmission duration could be expressed as [15]

$$
P_{i p}^{s}=\frac{\alpha_{1}}{T-\tau}\left(1-\exp \left(-\frac{T-\tau}{\alpha_{1}}\right)\right) .
$$

Moreover, in contrast with missed detection, another type of sensing errors that is referred to as false alarm, describes the situation that the spectrum is actually idle and wrongly detected as occupied by the PUs, which would also decrease the throughput and thus affect energy efficiency of cellular CRNs. Therefore, the optimal sensing time should be addressed in order to provide sufficient sensing accuracy and save the time as much as possible for data transmission in a unit frame.

\section{ENERgy EFficiency of Proposed Cellular CRN}

In this work, the SU employs energy detection spectrum sensing and transmits based on the decision made by the sensing phase. To control the interference to PU, the target detection probability $\overline{\mathscr{P}}_{d}$ should be guaranteed. With given sensing length and $\mathscr{\mathscr { P }}_{d}$, the probability of false alarm for the licensed channel under energy detection scheme is given by [8]:

$$
\mathscr{P}_{f a}(\tau)=\mathcal{Q}\left(\sqrt{2 \gamma+1} \mathcal{Q}^{-1}\left(\overline{\mathscr{P}}_{d}\right)+\sqrt{\tau f_{s}} \gamma\right)
$$

where $\tau$ represents the sensing time, $\gamma$ denotes the received SNR from the PU at the secondary detector on the licensed channel, $f_{s}$ represents the sampling frequency and $\mathcal{Q}(\cdot)$ stands for the complementary distribution function of the standard Gaussian.

Then, if the licensed channel is detected as idle $\left(\mathcal{H}_{0}\right)$, the SU would perform data transmission during the data transmission slot, whereas if the licensed band is sensed as occupied $\left(\mathcal{H}_{1}\right)$, the SU has to keep silent until the next frame. The instantaneous transmission rate of the $\mathrm{SU}$ on the licensed channel is denoted by $r_{0}$ when the channel is actual idle, and is denoted by $r_{1}$ when the channel is actual occupied by PU, which occurs when both the SU and the PU transmit on the same channel and interfere with each other. Based on the transmission interference links depicted in Fig. 2, $r_{0}$ and $r_{1}$ are respectively given by

$$
\begin{gathered}
r_{0}=\log _{2}\left(1+\frac{h_{s s} P_{s u}^{t}}{N_{0}}\right)=\log _{2}\left(1+\frac{P_{s u}^{0}}{N_{0}}\right), \\
r_{1}=\log _{2}\left(1+\frac{h_{s s} P_{s u}^{t}}{h_{p s} P_{p u}^{t}+N_{0}}\right)=\log _{2}\left(1+\frac{P_{s u}^{0}}{P_{p u}^{0}+N_{0}}\right),
\end{gathered}
$$

where $P_{s u}^{t}$ and $P_{p u}^{t}$ define the transmission power while $P_{s u}^{0}$ and $P_{p u}^{0}$ define the received power levels of secondary signal and primary signal at secondary receiver, respectively.

Based on the above analysis, the average interference to the PU and the average throughput of the SU could be expressed by (4) and (5), respectively (at the top of next page).

In order to formulate the energy efficiency problem of $\mathrm{SU}$, the power consumed in a frame duration should be addressed, which mainly contains three parts: electronic circuit consumption $P_{c}$, spectrum sensing consumption $P_{s}$, and the data transmission consumption on the licensed channel $P_{s u}^{t}$. The electronic circuit power $P_{c}$ is the average power assumption of device electronics, such as mixers, filters, and digital to analog converters, and it is almost fixed (given). Generally, the power used during spectrum sensing is much smaller compared to the data transmission power. The average total power consumed within a frame could be calculated as 


$$
\begin{gathered}
I=\frac{T-\tau}{T} \mathbb{E}\left\{P_{s u}^{0}\left(P\left(\mathcal{H}_{0}\right)\left(1-\mathscr{P}_{f a}\right) P_{p}^{s}+P\left(\mathcal{H}_{1}\right)\left(1-\mathscr{P}_{d}\right) P_{i p}^{s}\right)\right\} \\
R=\frac{T-\tau}{T} \mathbb{E}\left\{P\left(\mathcal{H}_{0}\right)\left(1-\mathscr{P}_{f a}\right)\left(r_{0}\left(1-P_{p}^{s}\right)+r_{1} P_{p}^{s}\right)+P\left(\mathcal{H}_{1}\right)\left(1-\mathscr{P}_{d}\right)\left(r_{0}\left(1-P_{i p}^{s}\right)+r_{1} P_{i p}^{s}\right)\right\} \\
J=\frac{(T-\tau)\left(P\left(\mathcal{H}_{0}\right)\left(1-\mathscr{P}_{f a}\right)\left(r_{0}\left(1-P_{p}^{s}\right)+r_{1} P_{p}^{s}\right)+P\left(\mathcal{H}_{1}\right)\left(1-\mathscr{P}_{d}\right)\left(r_{0}\left(1-P_{i p}^{s}\right)+r_{1} P_{i p}^{s}\right)\right)}{P_{c} T+P_{s} \tau+P_{s u}^{t}(T-\tau)\left(P\left(\mathcal{H}_{0}\right)\left(1-\mathscr{P}_{f a}\right)+P\left(\mathcal{H}_{1}\right)\left(1-\mathscr{P}_{d}\right)\right)}
\end{gathered}
$$

$$
E= \begin{cases}P_{c} T+P_{s} \tau, & \mathcal{H}_{1} \mid \mathcal{H}_{0} \text { or } \mathcal{H}_{1} \mid \mathcal{H}_{1} \\ P_{c} T+P_{s} \tau+P_{s u}^{t}(T-\tau), & \mathcal{H}_{0} \mid \mathcal{H}_{0} \text { or } \mathcal{H}_{0} \mid \mathcal{H}_{1}\end{cases}
$$

which could be expressed using conditional probability theory as

$$
\begin{aligned}
E= & P_{c} T+P_{s} \tau \\
& +P_{s u}^{t}(T-\tau)\left(P\left(\mathcal{H}_{0}\right)\left(1-\mathscr{P}_{f a}\right)+P\left(\mathcal{H}_{1}\right)\left(1-\mathscr{P}_{d}\right)\right) .
\end{aligned}
$$

The energy efficiency of the considered cellular CRN is defined as

$$
J=\frac{\text { Average Number of the Bits Received }}{\text { Average Total Energy Consumed }}=\frac{R T}{E}
$$

and it is measured in bits/Joule/Hz. By substituting (5) and (7) into (8), we can express the energy efficiency of SU in the considered cellular $\mathrm{CRN}$ with power control as (9) (at the top of this page).

Based on the derived energy efficiency formula for our proposed cellular CRN, the energy efficiency optimization problem, under the average interference constraint and total transmission power constraint can be formulated as follows

$$
\begin{array}{ll}
\text { maximize } & \mathbb{E}\{J\} \\
\text { subject to } & I \leq \bar{I}, R \geq \bar{R}, T>\tau>0 .
\end{array}
$$

where $\mathbb{E}\{J\}$ represents the expectation of $J$. Variable $\bar{I}$ denotes maximum acceptable interference for PU and $\bar{R}$ is the targeted throughput for the SU. It can be seen that (10) is exceptionally complex with complicated expressions for $J$ and $R$. The constraints $\bar{I}, \bar{R}$ are also not fixed due to different QoS of different wireless networks.

To explore the optimal sensing and frame slot duration, we take into account the concavity of the energy efficiency $J$ and throughput $R$. First, we examine the throughput which presents a simpler expression as energy efficiency. If we define $D=T-\tau$ which indicates the data transmission length, the expression of $R,(5)$, can be considered as function of $(\tau, D)$. Variables $\tau$ and $D$ are independent so that $P_{p}^{s}$ and $P_{i p}^{s}$ are as both functions of $D$ and have nothing to do with $\tau$. In this case, according to the Theorem 1 of [8], one can conclude that there exists an optimal sensing time which yields the maximum achievable throughput and $R$ is concave for the range of $\tau$ in which $\mathscr{P}_{f a} \leq 0.5$.

Proposition 1: Given the throughput of the $\mathrm{SU}$ as $R, R$ is not concave with respect to the frame length $T$.

Proof: See Appendix A.
In the sequel, as $J=\frac{R T}{E}$, the convexity of the derived energy efficiency with respect to $\tau$ and $T$ both depend on the value of multiplication factor $\frac{T}{E}$. In this case optimal values of $\tau$ and $T$ depend on the specific system characteristics in particular, on the assumed power consumption and primary traffic model. In order to find this value for the considered system conditions, different values of $\tau$ and $T$ have to be systematically tested to calculate the energy efficiency. That is, an exhaustive search has to be performed and it may require a certain amount of time and resources to obtain the optimum value. However, the values of $\tau$ and $T$ for the system is operational and it does not have to be calculated dynamically when the system is operational do not have to be computed dynamically if the PU traffic model does not change significantly. In other words, the optimal values of $\tau$ and $T$ can be obtained off-line only once for a particular system with stable PU traffic load and then they do not have to be recalculated again in a short term.

\section{Performance Analysis and Discussions}

In this section, we present and discuss the simulation results of the proposed cellular CRN environment with variable frame structure. We assume that all the mobile users including both the PU and SU are mutually independent and uniformly distributed in the typical cellular CRN. The probability density function (PDF) of the distribution of mobile users which are located at $\left(r_{d}, \theta_{d}\right)$ from its serving base station (BS) and can be given in polar coordinate as

$$
p\left(r_{d}, \theta_{d}\right)=\frac{r_{d}}{\pi R_{m}^{2}},
$$

where $R_{m}$ is the radius of macro-cell, $0 \leq r_{d} \leq R_{m}$ and $0 \leq \theta \leq 2 \pi$. To simulate a real channel environment, we consider a two slope path-loss model to obtain the mean received power as a function of distance between the mobile user and the respective serving BS [16]. Energy detection is employed for spectrum sensing. Simulated results are obtained using 10000 Monte-Carlo simulations. Major metrics such as energy efficiency and throughput are illustrated in per $\mathrm{Hz}$ basis. Unless otherwise stated, the values of the other parameters used are listed in Table I.

Fig. 3 consists of two sub-figures that illustrate energy efficiency of SU as a function of variable sensing length and variable frame length with fixed frame duration $T=100 \mathrm{~ms}$ and with fixed sensing duration $\tau=2 \mathrm{~ms}$, respectively. It can be clearly observed that there is an optimal sensing length with maximum achievable energy efficiency located at $1.8 \mathrm{~ms}$ while the optimal frame length value is located at $132 \mathrm{~ms}$. It is to note that the variable frame length is equivalent to a variable data transmission length if the sensing length is fixed. In addition, energy efficiency extended from the state-of-theart method [8] is illustrated by dashed lines for comparison. 

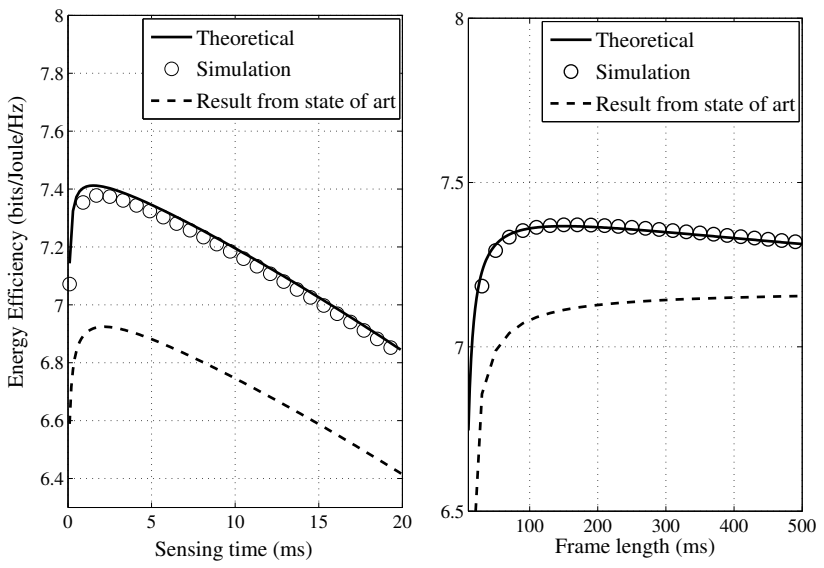

Fig. 3. Energy efficiency vs sensing time \& frame length compared with the results from [8].
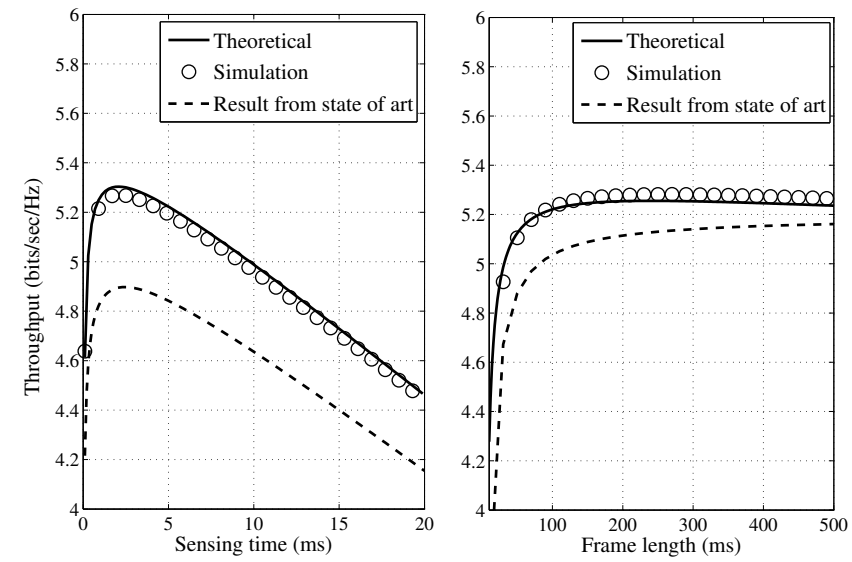

Fig. 4. Throughput vs sensing time \& frame length compared with the results from [8].

TABLE I

Simulation Parameter SetTings

\begin{tabular}{|c|c|}
\hline Narrowband bandwidth $(B)$ & $6 \mathrm{MHz}$ \\
\hline Noise PSD $\left(N_{0} / 2\right)$ & $-178 \mathrm{dBW} / \mathrm{Hz}$ \\
\hline Macro-cell radius $\left(r_{d}\right)$ & $500 \mathrm{~m}$ \\
\hline Circuit power $\left(P_{c}\right)$ & $200 \mathrm{~mW}$ \\
\hline Sensing power $\left(P_{s}\right)$ & $100 \mathrm{~mW}$ \\
\hline Transmission power $\left(P_{s u}^{t}\right)$ & $800 \mathrm{~mW}$ \\
\hline Worst-case received SNR from PU & $-15 \mathrm{~dB}$ \\
\hline Target detection probability $\left(\bar{P}_{d}\right)$ & $90 \%$ \\
\hline $\begin{array}{c}\text { Mean of traffic and idle duration } \\
\text { of PU }\left(\alpha_{1}, \alpha_{0}\right)\end{array}$ & $\begin{array}{c}\text { Voice Over Internet Protocol } \\
\text { (VOIP) }(352 \mathrm{~ms}, 650 \mathrm{~ms})\end{array}$ \\
\hline
\end{tabular}

It can be seen that our theoretical analysis based on derived expressions in Section III is much more accurate than that in [8] because unsynchronized traffic activity is considered which is a realistic feature in real network environments.

The achievable throughput results are also provided in Fig. 4 , which shows a very similar trend to the energy efficiency. However, compared with the optimal sensing value at $1.8 \mathrm{~ms}$ for maximum energy efficiency, there is still a diversity in terms of the optimal sensing lengths for maximum throughput, which is located at $2.2 \mathrm{~ms}$. A similar diversity between the
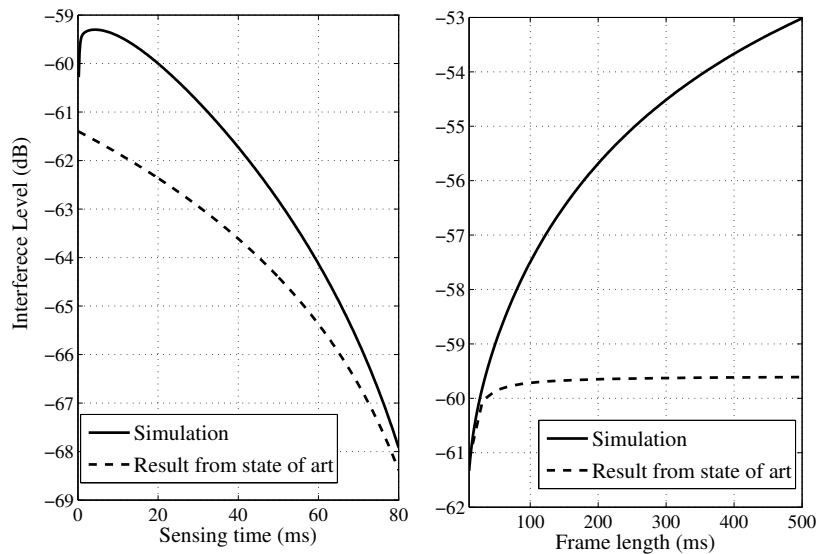

Fig. 5. Interference to PU vs sensing time \& frame length compared with the results from [8].

optimal frame lengths for maximum energy efficiency and throughput also exists at $132 \mathrm{~ms}$ and $236 \mathrm{~ms}$, respectively. Moreover, the derived throughput in [8] is also much lower than the values predicted by simulation results and the corresponding optimal sensing length with bias is $2.55 \mathrm{~ms}$ because of the same reason that ideal synchronization is assumed between the SU and PU traffic activities. It is observed that the our theoretical and simulated results are in perfect agreement for all cases.

Last but not least, the caused interference with the increase in sensing length and frame length is illustrated by Fig. 5 . There is a non-intuitive result shown in Fig. 5. When the sensing time is very short such as less than $1.5 \mathrm{~ms}$, interference increases with the increase in sensing time. This is because the target $\overline{\mathscr{P}}_{d}$ is fixed as 0.9 , and as $\tau$ increases, $\mathscr{P}_{f a}$ decreases which leads to more cognitive secondary transmission and thus higher $P_{p}^{s}$. However, the level of interference decreases with a further significant increase in spectrum sensing time $\tau$ since $\mathscr{P}_{f a}$ will now be a rather small constant under such condition. Therefore, allocating more time for spectrum sensing with a fixed frame length such as $100 \mathrm{~ms}$, leads to an accurate spectrum sensing and thereby reduces the interference with a fixed frame length. While the result pertaining to various frame durations with fixed sensing length is much more intuitive, it can be justified through the fact that longer frame durations lead to a much higher $P_{p}^{s}$ which causes additional interference. Additionally, consistent with previous energy efficiency and throughout results, the interference levels extended from [8] are significantly underestimated compared to our simulation results.

\section{CONCLUSIONS}

In this paper, we analyzed the energy efficiency of the $\mathrm{SU}$ in a proposed cellular CRN based on the frame structure which consists of variable sensing and data transmission slots. The achievable bits per Joule of the SU has been analytically expressed under a realistic VOIP traffic model. The impact of variable sensing and frame lengths, on the achievable energy efficiency and interference have been critically illustrated with energy detection spectrum sensing. Moreover, the SU's achievable throughput is also provided for comparison. The analysis of the diversity between the optimal frame structures with achievable maximum energy efficiency and throughput of the $\mathrm{SU}$ is useful to determine the future required cognitive frame structure for cellular CRN. The accuracy of our analysis 
has been verified by both simulations and comparisons with relevant state of art. The proper frame structure should also be adaptively chosen to achieve the required performance of the SU and at the same time to further reduce the interference to the legal PU.

\section{ApPENDIX A: PROOF OF PROPOSITION 1}

The first partial derivative of variable $R$ with respect to $T$ can be expressed as

$$
\begin{aligned}
& R(T)=\frac{\tau}{T^{2}}\left\{P\left(\mathcal{H}_{0}\right)\left(1-\mathscr{P}_{f a}\right)\left(r_{0}\left(1-P_{p}^{s}\right)+r_{1} P_{p}^{s}\right)\right. \\
& \left.\quad+P\left(\mathcal{H}_{1}\right)\left(1-\mathscr{P}_{d}\right)\left(r_{0}\left(1-P_{i p}^{s}\right)+r_{1} P_{i p}^{s}\right)\right\} \\
& \quad+\frac{T-\tau}{T}\left\{P\left(\mathcal{H}_{0}\right)\left(1-\mathscr{P}_{f a}\right) \times\left(r_{0}\left(-\frac{\partial P_{p}^{s}}{\partial T}\right)+r_{1} \frac{\partial P_{p}^{s}}{\partial T}\right)\right. \\
& \left.\quad+P\left(\mathcal{H}_{1}\right)\left(1-\mathscr{P}_{d}\right)\left(r_{0}\left(-\frac{\partial P_{i p}^{s}}{\partial T}\right)+r_{1} \frac{\partial P_{i p}^{s}}{\partial T}\right)\right\} .
\end{aligned}
$$

With series expansion of exponential function $e^{x}=1+\frac{x}{1 !}+$ $\frac{x^{2}}{2 !}+\frac{x^{3}}{3 !}+\ldots$ and when $T$ approaches $\tau$ and $+\infty$, the limits of $P_{p}^{s}$ and $P_{i p}^{s}$ are equal to as

$$
\begin{aligned}
& \lim _{T \rightarrow \tau} P_{p}^{s}=\lim _{T \rightarrow \tau}\left(1-\frac{\alpha_{0}}{T-\tau}\left(1-\exp \left(-\frac{T-\tau}{\alpha_{0}}\right)\right)\right) \\
& =0 \text {, } \\
& \lim _{T \rightarrow \tau} P_{i p}^{s}=\lim _{T \rightarrow \tau} \frac{\alpha_{1}}{T-\tau}\left(1-\exp \left(-\frac{T-\tau}{\alpha_{1}}\right)\right)=1 \text {, } \\
& \lim _{T \rightarrow+\infty} P_{p}^{s}=\lim _{T \rightarrow+\infty}\left(1-\frac{\alpha_{0}}{T-\tau}\left(1-\exp \left(-\frac{T-\tau}{\alpha_{0}}\right)\right)\right) \\
& =1 \text {, } \\
& \lim _{T \rightarrow+\infty} P_{i p}^{s}=\lim _{T \rightarrow+\infty} \frac{\alpha_{1}}{T-\tau}\left(1-\exp \left(-\frac{T-\tau}{\alpha_{1}}\right)\right)=0,
\end{aligned}
$$

respectively.

Similarly, the limits of $\frac{\partial P_{p}^{s}}{\partial T}(T-\tau)$ and $\frac{\partial P_{i p}^{s}}{\partial T}(T-\tau)$ when $T$ approaches $\tau$ and $+\infty$ can be expressed as

$$
\begin{aligned}
\lim _{T \rightarrow \tau} \frac{\partial P_{p}^{s}}{\partial T}(T-\tau)= & \lim _{T \rightarrow \tau} \frac{\alpha_{0}}{(T-\tau)}\left(1-\exp \left(-\frac{T-\tau}{\alpha_{0}}\right)\right) \\
& -\lim _{T \rightarrow \tau} \exp \left(-\frac{T-\tau}{\alpha_{0}}\right)=0, \quad \text { (A.6) } \\
\lim _{T \rightarrow \tau} \frac{\partial P_{i p}^{s}}{\partial T}(T-\tau)= & \lim _{T \rightarrow \tau} \frac{-\alpha_{1}}{(T-\tau)}\left(1-\exp \left(-\frac{T-\tau}{\alpha_{1}}\right)\right) \\
& +\lim _{T \rightarrow \tau} \exp \left(-\frac{T-\tau}{\alpha_{1}}\right)=0, \quad \text { (A.7) } \\
\lim _{T \rightarrow+\infty} \frac{\partial P_{p}^{s}}{\partial T}(T-\tau)= & \lim _{T \rightarrow+\infty} \frac{\partial P_{i p}^{s}}{\partial T}(T-\tau)=0,
\end{aligned}
$$

respectively.

By substituting the equations (A.2) - (A.8) into (A.1), the limits of $R(T)$, when $T$ approaches $\tau$ and $+\infty$, can be finally expressed as

$$
\begin{aligned}
\lim _{T \rightarrow \tau} R(T)= & \frac{1}{\tau}\left(P\left(\mathcal{H}_{0}\right)\left(1-\mathscr{P}_{f a}\right) r_{0}+P\left(\mathcal{H}_{1}\right)\left(1-\mathscr{P}_{d}\right) r_{1}\right) \\
& >0, \\
\lim _{T \rightarrow+\infty} R(T)= & 0\left(P\left(\mathcal{H}_{0}\right)\left(1-\mathscr{P}_{f a}\right) r_{1}+P\left(\mathcal{H}_{1}\right)\left(1-\mathscr{P}_{d}\right) r_{0}\right) \\
& =0,
\end{aligned}
$$

respectively.

\section{REFERENCES}

[1] F. C. Commission, "Spectrum policy task force report, fcc 02-155," Tech. Rep., 2002.

[2] J. Mitola and G. Q. Maguire, "Cognitive radio: making software radios more personal," Personal Communications, IEEE, vol. 6, no. 4, pp. 13-18, Aug. 1999.

[3] S. Haykin, "Cognitive radio: brain-empowered wireless communications," Selected Areas in Communications, IEEE Journal on, vol. 23, no. 2, pp. 201-220, Feb. 2005.

[4] Q. Zhao and A. Swami, "A survey of dynamic spectrum access: Signal processing and networking perspectives," in Acoustics, Speech and Signal Processing, 2007. ICASSP 2007. IEEE International Conference on, vol. 4, Apr. 2007, pp. 1349-1352.

[5] I. F. Akyildiz, W.-Y. Lee, M. Vuran, and S. Mohanty, "A survey on spectrum management in cognitive radio networks," Communications Magazine, IEEE, vol. 46, no. 4, pp. 40-48, Apr. 2008.

[6] D. Pamlin and K. Szomolanyi, "Saving the climate @ the speed of light-first roadmap for reduced co2 emissions in the eu and beyond," World Wildlife Fund and European Telecoms Network Operators Association, 2007.

[7] C. Anderson, D. Freeman, I. James, A. Johnston and S. Ljung, Mobile Media and Applications, From concept to Cash: Successful Service Creation and Launch. Wiley, 2006.

[8] Y.-C. Liang, Y. Zeng, E. C. Y. Peh, and A. T. Hoang, "Sensing-throughput tradeoff for cognitive radio networks," Wireless Communications, IEEE Transactions on, vol. 7, no. 4, pp. 1326 -1337, Apr. 2008.

[9] Y. Pei, Y.-C. Liang, K. C. Teh, and K. H. Li, "Sensingthroughput tradeoff for cognitive radio networks: A multiple-channel scenario," in Personal, Indoor and Mobile Radio Communications (PIMRC), 2009 IEEE 20th International Symposium on, Sep. 2009, pp. 1257-1261.

[10] S. Zarrin and T. J. Lim, "Throughput-sensing tradeoff of cognitive radio networks based on quickest sensing," in Communications (ICC), 2011 IEEE International Conference on, June 2011, pp. 1-5.

[11] Y. Pei, Y.-C. Liang, K. C. Teh, and K. H. Li, "Energyefficient design of sequential channel sensing in cognitive radio networks: Optimal sensing strategy, power allocation, and sensing order," Selected Areas in Communications, IEEE Journal on, vol. 29, no. 8, pp. 1648-1659, Sep. 2011

[12] L. Li, X. Zhou, H. Xu, G. Y. Li, D. Wang, and A. Soong, "Energy-efficient transmission in cognitive radio networks," in Consumer Communications and Networking Conference (CCNC), 2010 7th IEEE, Jan. 2010, pp. 1-5.

[13] F. F. Digham, M.-S. Alouini, and M. K. Simon, "On the energy detection of unknown signals over fading channels," Communications, IEEE Transactions on, vol. 55, no. 1, pp. 21-24, 2007.

[14] Andrea Goldsmith, Wireless Communications. Cambridge University Press, New York, 2003.

[15] W. Tang, M. Z. Shakir, M. A. Imran, R. Tafazolli, and M.-S. Alouini, "Throughput analysis for cognitive radio networks with multiple primary users and imperfect spectrum sensing," Communications, IET, vol. 6, no. 17, pp. 2787-2795, 2012.

[16] M.-S. Alouini and A. J. Goldsmith, "Area spectral efficiency of cellular mobile radio systems," Vehicular Technology, IEEE Transactions on, vol. 48, no. 4, pp. 1047-1066, 1999. 